\title{
Multi-Metric Route Cost Based Routing in Wireless Multimedia AD-HOC Networks
}

\author{
Parvinder Singh, Rajeshwar Singh
}

\begin{abstract}
To improve the quality of life, bigger cities in the world addressing their problems by adopting latest technology. IOT connect physical world to the internet and such concept is adopted by bigger cities having large population growth rate. Wireless multimedia sensor network helps to monitor various parts of a city without use of cables. This network becomes the primary component of every smart city due to its surveillance and monitoring capability In contrast with conventional sensor networks, multimedia sensor nodes are equipped with cameras which acquire, process, compress and transmit information about an event happing at some remote area in the form of an image or video. However, like conventional WSNs this network is having multiple constrained and new metric needs to be considered to improve network life time and Quality of service parameters. Multiple metric based route cost function is defined and routing mechanism based upon this cost function is proposed. Since each multimedia sensor node is battery operated and connected through low power wireless links. This work helps to find an optimal path having minimum energy consumption, maximum link reliability, minimum number of hops and minimum routing table magnitude. A cost function considers constraints of wireless multimedia sensor networks. It satisfies desirable network requirements and improves network life time through efficient routing. The proposed algorithm is tested, simulated and compared with previous routing algorithms.
\end{abstract}

Index Terms: IEEE 802.15.4, Wireless multimedia sensor networks, Reliability, Storage constraints, Hop-metric, Route cost function

\section{INTRODUCTION}

Wireless multimedia sensor networks (WMSN) provide more information than traditional WSNs. But, at the same time nodes of WMSNs consumes more power and wireless links need more bandwidth to transmit bulk amount of image and video data as compared to scalar sensor nodes. Recent work [1] proposed the design approaches of WMSN, first approach consider homogenous nodes having surveillance capability. In the second approach heterogeneous network can be designed having two types of nodes. Work having more computational complexity can be distributed to some highly capable devices.A multi-tier WMSN is more suitable for real-time video surveillance applications.. Second design approach addresses energy optimization as well as image improvement in a battery-operated wireless multimedia surveillance scenario. The work in [2] states a new sample based transmission approach that utilizes haar wavelet transform. A parking lot in a smart city can be monitored and its images help to send status occupancy. This technique demands fewer measurements and supports energy efficient parking monitoring system using WVSNs Information

Revised Manuscript Received on June 25, 2019

Parvinder Singh, ECE Departent, PTU, Jalandhar, India.

Dr Rajeshwar Singh, ECE Department, Director, DKTGI, Chhokran, Rahon, SBS Nagar, Punjab, India

gathering can be made more efficient if the sensors are deployed in relevance to the sink [3]. Position of sensors always play an important role in low power battery operated WMSNs. This approach tested with two different scenarios: one is configuration scenario, with sensors deployment in different position and relevance previously defined. There are multiple smart lighting projects having sensors are employed to assist lighting system [4], [5], [6], [7] or in smart sensor buildings [8], They basically exploit the luminance using LED diode technology as it allows dimming control [5].

These systems basically deploy sensors to observe environmental light, power efficiency, and the presence of people, based on which they can control the system in order to improve the quality and the energy-efficiency of the intelligent service [8], [9]. Another important area of WMSNs for smart cities is outdoor and indoor environmental monitoring. In traditional techniques it is done by a large quantity of costly, low-precision sensors; while WMSNs offer an small number of low-cost, high-precision units [10]. Cloud based module prototype in [11].

For wireless adhoc networks based upon low-data rate wireless technologies, such as Bluetooth, IEEE 802.15.4 protocol [12], large packet data generated by video sensors may not be received due to low data rate. For dense WMSN with large number of active multimedia nodes, IEEE 802.11 [13] networks considered as reasonable solution. Unlike traditional WSNs, the WMSN contains multimedia content which adversely affect the routing performance, i.e. when a node is continually sensing, routes having sensor nodes of high residual energy will be considered[14]. Fuzzy based power saving approach is considered in [15], which consider switching off APs when traffic load is low.

The efficient relay of multimedia video frames on a routing protocol, which determine sturdy and energy efficient path, and to extend different levels of Quality-of-Experience (QoE)/Quality of-Service (QoS)) assurance for multimedia. However, it is a testing issue due to narrow resources [16].

\section{RELATED WORK}

\section{A. ETRT Based Routing}

Recent work considers multiple metric while designing routing decision [17] in the low power IOT networks. Transmission range of nodes can be optimized by estimating ETRT value and it further optimized the network performance in terms of energy efficiency and QOS provision. However, authors do not consider other constraints like storage capability, computational capability etc. Cross- layer models are also complex to understand and hidden energy consumption always takes place while exchanging of crosslayer information.

\section{B. Fuzzy based EE approach}

The work in [18] consider wireless multimedia sensor networks connected through

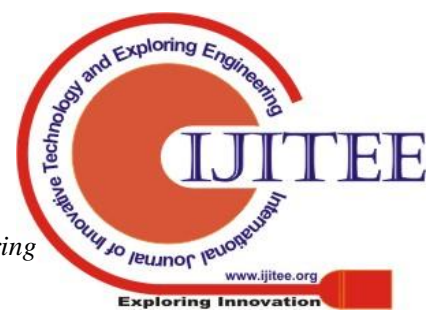


Wi-Fi standard. It saves energy by switching-off access-points through fuzzy controller. This approach basically considers energy efficiency and do not take into account other quality of service parameters.

\section{Smart city relevance based approach}

Wireless multimedia sensor networks may become permanent member of smart city applications due to its unique characteristics [19]. Authors present an innovative technique of positioning of sinks close to source nodes which produce high amount of data. Big cities always demands traffic control through realistic approach so, this technique can efficiently handle traffic generated zones.

\section{PROPOSED PROTOCOL ARCHITECTURE}

The proposed protocol architecture is shown in figure 1. It consist of route discovery message processing unit, route cost estimation unit, table management system, timing unit.

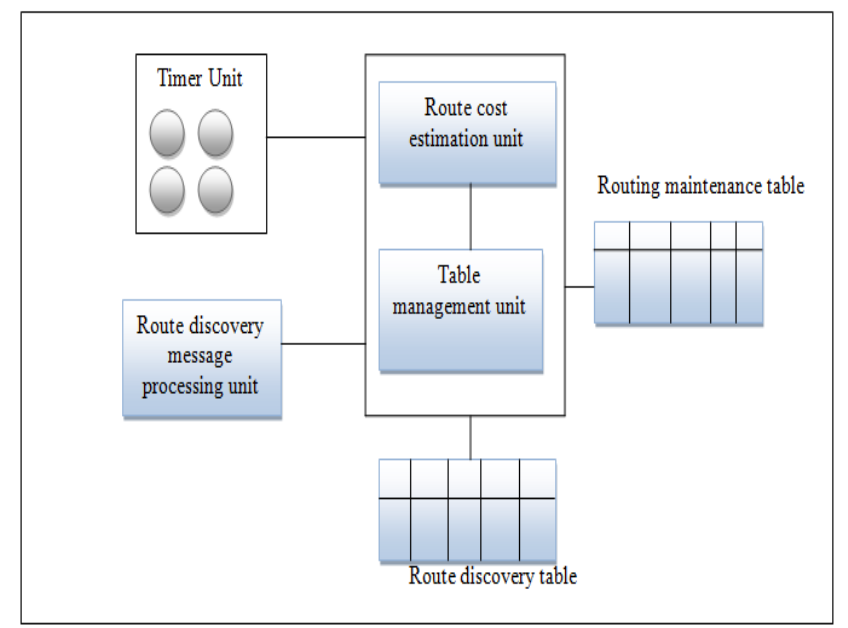

Figure 1. Proposed protocol architecture

The discovery message processing unit processes and forward the route discovery messages and also other cross layer messages from MAC layer and application layer. The table management system maintains the operation of route discovery and route maintenance table.

During the discovery of route, RREQ is generated and sent along neighboring nodes. The nodes reply through RREP and accordingly route discovery table generated. The route cost estimation unit outlines route cost function for the route setup and select the most suitable route through route cost function.

\section{ROUTE COST ESTIMATION}

Multimedia wireless sensor network includes nodes having multiple constraints in terms of memory, processor capability and energy as compare to traditional WSNs. Therefore, routing protocols of wireless multimedia sensor networks need new metrics for path selection. Considering the limitations of multimedia nodes, protocols should be designed with new metrics while calculation of routes. In this work, we examine the following metrics for real time network platforms.

\section{A. Expected energy cost}

This is both node level and link level metric for energy constraint and its very important metric as far as energy efficiency is concerned. At node level it includes transmission power, reception power, sensing power and processing power for a fixed size data packet while at link level the channel condition variation effect retransmission.

\section{B. Routing Table Magnitude}

This is node level metric for storage constraint.

\section{Link reliability indicator (LRI) level}

This is a link level metric considering unreliable channel constraint, which measure the channel reliability using a numerical value. This metric affects the average throughput. Link reliability consideration improves energy efficiency and QOS parameters in constrained based networks. Its value vary upto 7 in standard presented in [20].

\section{Hop-Count metric}

This metric describe the number of hops along a route and it affects the routing latency.

We proposed a new route cost function in equation (1) and by having prior knowledge of above routing metrics and apply them to standard AODV protocol.

$$
\begin{gathered}
\mathrm{R}^{*}=\arg \min \left\{\mu_{e} R_{e}+\mu_{n} R_{n}+\mu_{l} R_{l}+\mu_{h} R_{l}\right\} \ldots \ldots . \\
\mu_{e}+\mu_{n}+\mu_{l}+\mu_{h}=1 \\
R_{e}=\arg \sum \min \left\{1, \quad \frac{\text { CONSUMRD_ENERGY (e) }}{\text { MAX_TOTAL_ENERGY }}\right\} \\
R_{n}=\arg \sum \min \left\{1, \frac{\text { REFR_ENT(n) }}{\text { ROUTE TABLE SIZE }}\right\} \\
R_{1}=\arg \sum \min \left\{0,1-\frac{\text { LRI_ENT(1) }}{\text { MAXIMUM LEVEL }}\right\} \\
R_{h}=\text { HOP_ENT }
\end{gathered}
$$

$\mathrm{R}^{*}$ is the route selected while delivering control commands or data to wireless multimedia sensor networks and $\mu$ represents the weight for optimizing the route cost to prioritize the requirement of network and it is described on weighting sum method. The path having lowest energy cost, highest reliability, lowest hop count and lowest routing table magnitude decidedly selected for data transmission. Figure 2 shows the optimum path selected by the proposed protocol.

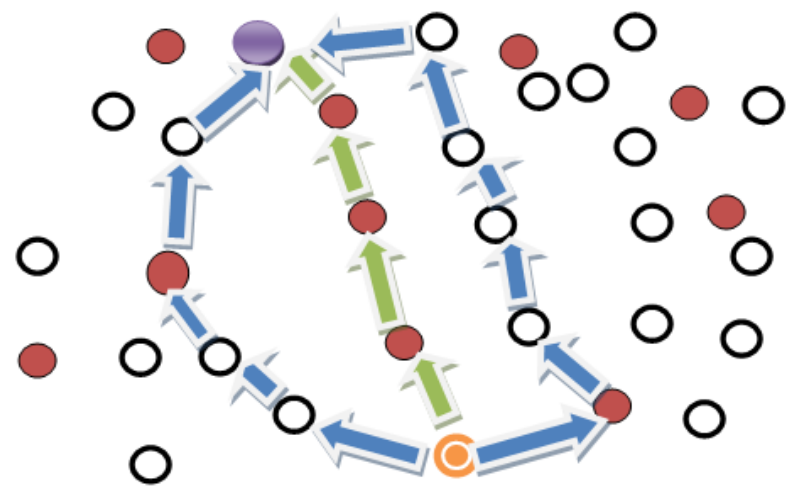
Link in optimum path Link in other alternative paths 


\section{Figure 2. Optimum path selection in proposed routing} protocol

\section{RESULTS AND DISCUSSIONS}

To describe the performance of our proposed routing technique, different scenarios are considered. 100 nodes are distributed in $1800 \mathrm{~m} * 1800 \mathrm{~m}$ network area and nodes are distributed randomly. For each node initial energy level set to 150 joules and transmission range set to $300 \mathrm{~m}$. Two contrasting scenarios are considered to see the impact on the proposed protocol. First we vary the size of video frame and consider speed of visual sensor node fixed. In the subsequent scenario speed of visual sensor node is changed and video frame size kept fixed.

\section{A. Table 1 Simulation Parameters}

\begin{tabular}{|c|c|c|}
\hline Variable & Value & units \\
\hline Nodes & 100 & Node \\
\hline Frame size & $32,64,128,256,512,1024$ & Byte \\
\hline Simulation Area & $1800 * 1800$ & Meter square \\
\hline Mobility Model & Random waypoint & \\
\hline Traffic Type & CBR & \\
\hline Starting Energy & 150 & Joules \\
\hline Transmission power & 0.1 & Joules \\
\hline Receive power & 0.07 & Joules \\
\hline Sleep power & 0.001 & Joules \\
\hline
\end{tabular}

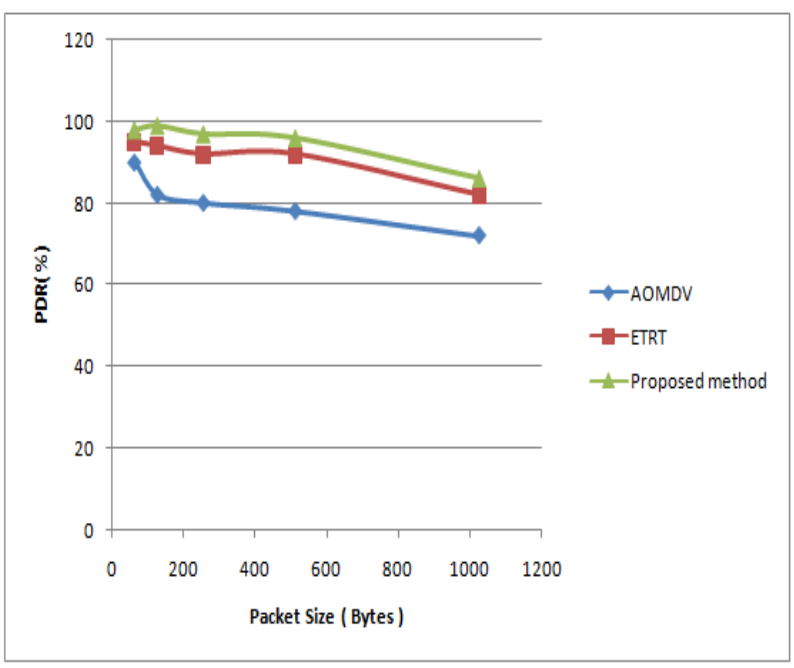

Figure 3. PDR vs Packet Size

Figure 3 shows the packet delivery ratio variation for AOMDV, ETRT and proposed routing scheme with increase in packet size.With increase in size of packet, the packet delivery ratio decreases but the proposed technique curve slope shows high PDR value and it maintains high PDR as compared to previous technique.

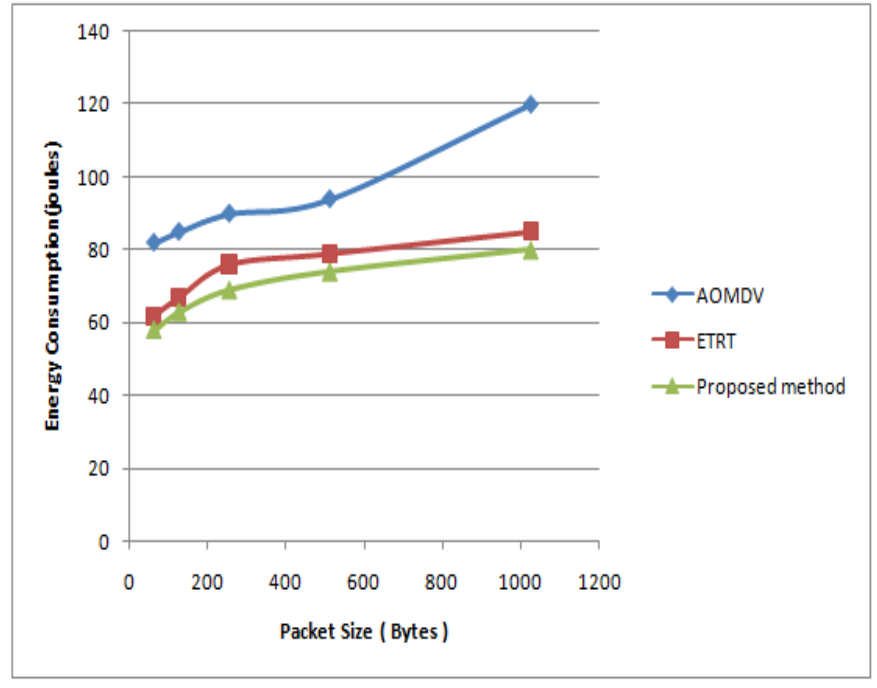

Figure.4 Energy consumption vs Packet Size

Figure 4 shows energy consumption for AOMDV, ETRT and proposed routing scheme with increase in packet size. Due to difference in the routing mechanism of proposed method the energy consumption is lesser in proposed protocol as compared to other two techniques.

\section{CONCLUSION}

Multimedia sensors are battery operated and are connected through wireless links, which motivate to consider the importance of power efficiency. So, power saving and QoS are the utmost important issues needs to be addressed in wireless multimedia sensor networks. Power consumption not only depends upon transmission power but it needs to consider other factor like reliability of links and hop count to achieve optimum minimum power while maintaining quality of service parameters. To solve these issues we defined routing mechanism through route cost function considering hop count, link energy cost, link reliability and routing table magnitude as metrics. The optimization of these parameters can be achieved by adjusting associated weights. The aim is to select a path that includes more reliable links and minimum number of hops while conserve energy. Our results prove the suitability of this technique in dense wireless multimedia sensor networks. The proposed method proves better performance than standard AODV and ETRT techniques.

\section{REFRENCES}

[1] Tenager mekonnen, pawani porambage, erkki harjul and mika ylianttila "Energy Consumption Analysis of High Quality Multi-Tier Wireless Multimedia Sensor Network" IEEE Access, 2017, vol. 5.

[2] R. Monika \& R. Hemalatha and S. Radha, "Energy efficient surveillance system using WVSN with reweighted sampling in modified fast Haar wavelet transform domain" Multimed Tools Appl (2018).

[3] Joao Paulo,Just Peixoto and Daniel G. Costa, “ Wireless visual sensor networks for smart city applications: A relevance-based approach for multiple sinks mobility" Future Generation Computer Systems 76 (2017) 51-62.

[4] G. Shahzad, H. Yang, A. W. Ahmad, and C. Lee, "Energy-efficient intelligent street lighting system using traffic-adaptive control," IEEE Sensors Journal, vol. 16, no. 13, 2016, pp. 5397-5405. 
[5] A. Kovacs, R. B 'atai, B. Cs. Cs ' aji, P. Dud ' as, B. H'ay, G. Pedone, ' T. Rev' esz, and J. V' ancza, "Intelligent control for energy-positive street ' lighting," Energy, vol. 114, pp. 40-51, 2016

[6] A. Lavric, V. Popa, and S. Sfichi, "Street lighting control system based on large-scale WSN: A step towards a smart city," in 2014 International Conference and Exposition on Electrical and Power Engineering (EPE). IEEE, 2014, pp. 673-676.

[7] M. Mahoor, F. R. Salmasi, and T. A. Najafabadi, "A hierarchical smart street lighting system with brute-force energy optimization," IEEE Sensors Journal, vol. 17, no. 9, 2017, pp. 2871-2879

[8] F. Viani, A. Polo, P. Garofalo, N. Anselmi, M. Salucci, and E. Giarola, "Evolutionary optimization applied to wireless smart lighting in energyefficient museums," IEEE Sensors Journal, vol. 17, no. 5, 2017, pp. $1213-1214$

[9] F. Viani, A. Polo, F. Robol, E. Giarola, and A. Ferro, "Experimental validation of a wireless distributed system for smart public lighting management," in IEEE International Smart Cities Conference (ISC2), Trento, Italy, September 12-15, 2016, pp. 678-683.

[10] L. M. Oliveira and J. J. Rodrigues, "Wireless sensor networks: A survey on environmental monitoring." Journal of Communications, vol. 6, no. 2, 2011, pp. 143-151.

[11] Balazs Cs.aji, Zsolt Kemeny, Gianfranco Pedone, Andr Kuti, J'ozsef Vancza "Wireless Multi-Sensor Networks for Smart Cities: A Prototype System with Statistical Data Analysis" IEEE sensors journal.

[12] A. Monsalve, H.L. Vu, Q.B. Vo, "Optimal designs for IEEE 802.15.4 wireless sensor networks", Wirel. Commun. Mob. Comput. 13 (18) (2013) 1681-1692.

[13]V. Vora, T. Brown, "High rate video streaming over $802.11 \mathrm{n}$ in dense wi-fi environments", in: IEEE Conference on Local Computer Networks, 2010, pp. 1054-1061.

[14]Pan Zhaoqing, Zhang Yun, Kwong Sam, "Efficient motion and disparity estimation optimization for low complexity multiview video coding" IEEE Trans. Broadcast. 61 (2) , 2015, 166-176.

[15] Mario Collottaa,Giovanni Paua,Daniel G. Costa "A fuzzy-based approach for energy-efficient Wi-Fi communications in dense wireless multimedia sensor networks" Computer Networks 134 (2018) $127-139$.

[16] Hang Shen, Guangwei Bai, "Routing in wireless multimedia sensor networks: A survey and challenges ahead", Journal of Network and Computer Applications 71 (2016) 30-49.

[17] Sarwesh P, N. Shekar V. Shet, K. Chandrasekaran "ETRT - Cross layer model for optimizing transmission range of nodes in low power wireless networks - An Internet of Things Perspective" Physical Communication 29 (2018) 307-318

[18] Mario Collotta, Giovanni Pa , Daniel G. Costa “ A fuzzy-based approach for energy-efficient Wi-Fi communications in dense wireless multimedia sensor networks" Computer Networks 134 (2018) 127-13.

[19] Joao Paulo, Just Peixoto, Daniel G. Costa "Wireless visual sensor networks for smart city applications:A relevance-based approach for multiple sinks mobility" Future Generation Computer Systems 76 (2017) 51-62.

\section{AUTHORS PROFILE}

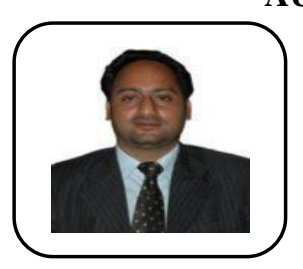

Parvinder Singh is presently working as Associate professor in RIEIT, Railmajra, SBS Nagar, Punjab. He is pursuing his Ph.D from Punjab Technical University, Jalandhar in Electronics and Communication Engg department. He did his masters degree in Electronics and communication from DAVIET, Jalandhar. He has more than 13 years of teaching experience. His current research interests include Wireless Adhoc Networks (Energy, Security, Routing), Wireless Sensor Network, Mobile Ad hoc Network Routing, Wireless Commincations.

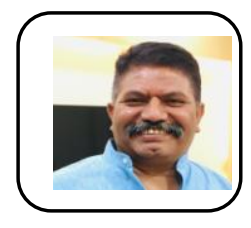

Prof. (Dr.) Rajeshwar Singh is presently working as Director, Doaba Khalsa Trust Group of Institutions, SBS Nagar, Punjab. He received his Ph.D. Engineering degree from Department of Electronics and Communication Engineering, Faculty of Engineering, BIT Sindri, Dhanbad, Jharkhand. His Master of Engineering degree is in Electronics and Communication Engineering with specialization in Digital Systems from Motilal Nehru Regional Engineering College (currently NIT), Allahabad, U.P. He received his AMIE (India) degree in 1992 from The Institutions of Engineers, Calcutta. He has also received Master of Business Administration (MBA) in Information Technology from MD University, Rohtak, Haryana.
He has more than 26 years of experience in teaching and industry. He has published more than 100 papers in national and international journals / conferences of repute. He has also published four books in the field of Engineering and Technology. His current research interests include Swarm Intelligence based Optimization (Energy, Security, Routing), Wireless Sensor Network, intellectual information technology, Mobile Ad hoc Network Routing, Coding theory, cryptography, e-governance and Educational Planning. $\mathrm{He}$ is member of ISTE, AMIE and various engineering organizations. He is also editor-in-chief/editor/reviewer of various national and International Journals. 